\title{
Desarrollo de una herramienta de software para la gestión del mantenimiento de infraestructura en el SENA regional Antioquia
}

\section{Development of a software tool for the management of infrastructure maintenance in the SENA Antioquia regional}

\author{
Juan David Vahos a, Ana Alexandra Pino-Martínez b \& John Jairo Castro Maldonado c \\ a Instructor, Centro de Servicios y Gestión Empresarial - CESGE, Grupo GIGAT, Servicio Nacional de Aprendizaje SENA, \\ Medellín-Colombia, jvahosmo@sena.edu.co \\ ${ }^{b}$ Investigadora SENNOVA, Centro de Servicios y Gestión Empresarial - CESGE, Grupo GIGAT, Servicio Nacional de \\ Aprendizaje SENA, Medellin-Colombia, apinom@sena.edu.co \\ c Instructor, Centro de Servicios y Gestión Empresarial - CESGE, Grupo GIGAT, Servicio Nacional de Aprendizaje SENA, \\ Medellín-Colombia, jcastrom@misena.edu.co
}

\section{Resumen}

Recibido: abril 9, 2019. Aceptado: noviembre 30, 2019.

En la actualidad, la informática ha ayudado en la implementación de soluciones para la gestión de mantenimiento de equipos, facilitando la realización de tareas y reduciendo los costes de forma considerable. En este sentido, se ha buscado el desarrollo de una herramienta de software para dar solución al problema de gestión de los procesos de mantenimiento de infraestructura del Servicio Nacional de Aprendizaje - SENA Regional Antioquia, ya que anteriormente tales servicios se solicitaban a través de trámites engorrosos, usando formatos de Excel, documentos de Word y el correo electrónico continuamente, dificultando la trazabilidad, y la asignación óptima de los recursos humanos y técnicos a la hora de realizar los correctivos pertinentes. La aplicación web de gestión desarrollada minimiza los tiempos improductivos del personal, permitiendo a la organización optimizar recursos humanos, económicos y ambientales al mejorar la atención de aquellos problemas y requerimientos que pueden prevenirse mediante un mantenimiento efectivo y adecuado. Además, esta herramienta aporta al desarrollo sostenible por medio de la minimización del papel, dado que a través de alertas y notificaciones en línea evita la impresión física de los formatos para cada fase del proceso y simplifica las estadísticas, pues se pueden conocer con certeza las operaciones realizadas en un lapso de tiempo determinado; un control y seguimiento preciso de estos procesos es vital para cualquier empresa u organización que posea bienes o inmuebles que requieren mantenimiento periódico.

Palabras Clave: Mantenimiento, procesos, software, gestión, trazabilidad.

\begin{abstract}
This article describes the development of a software tool to solve the problem of management for the processes of infrastructure maintenance of the National Training Service - SENA Regional Antioquia. Previously, those maintenance tasks were requested through cumbersome procedures using Excel formats, Word documents, and e-mail messages. The sheer quantity of transactions and communications involved difficulted the traceability of the tasks and the optimal allocation of human and technical resources to perform the corrective measures. The web management tool developed minimizes downtime of the staff, allowing the organization to optimize human resources. Also, the software affects economic and environmental factors through effective and appropriate maintenance management of equipment. Besides, this tool contributes to sustainable development minimizing the use of paper by the offering of alerts and notifications online and preventing the physical impression of the formats for each stage of the process. Developed software also simplifies the calculation of statistics, since it can be known with certainty the number of executed operations a given time. The precise tracking and control of maintenance processes are fundamental for management in any organization or company owning goods or properties in need of periodic maintenance.
\end{abstract}

Keywords: Maintenance, processes, software, management, traceability.

\section{INTRODUCCIÓN}

En general, las empresas tienen como fin la gestión y optimización de sus recursos para producir los bienes y servicios que permitan la continuidad y el crecimiento al negocio. El éxito en las actividades empresariales es altamente dependiente de todas las partes que conforman la estructura organizacional [1], y en particular, la gestión de mantenimiento desempeña un rol preponderante. La base de la ingeniería de mantenimiento es la

\section{Citar como:}

J. Vahos, A. Pino, J. Castro. "Desarrollo de una herramienta de software para la gestión del mantenimiento de infraestructura en el SENA regional Antioquia". Revista CINTEX, Vol. 24(1), pp. 13-19. 2019. 
mejora continua a través de la incorporación de inteligencia, análisis y conocimiento que apoyen la toma de decisiones de mantenimiento, buscando a favorecer tanto el resultado económico como el operacional [2].

El mundo actual depende en gran medida de la tecnología y la automatización. A medida que avanza la tecnología, podemos hacer más con las máquinas, pero también dependemos más de ellas. En muchas industrias, el equipo requerido para producir bienes y servicios es costoso de poseer, operar y mantener, y las fallas incluso en una sola pieza de equipo pueden detener una producción completa. Además de las pérdidas directas de producción, el tiempo de inactividad causado por una falla del equipo puede dañar la reputación de la empresa y, en algunos casos, generar multas y sanciones. La falla del equipo también puede poner en peligro la salud y la seguridad. Estas cosas hacen que mantener el equipo correctamente sea una actividad clave para las empresas $\mathrm{y}$, en algunas industrias, las empresas gastan hasta el $50 \%$ de sus costos operativos en mantenimiento. En el entorno competitivo de hoy, comprender el costo total de mantenimiento y optimizar estos costos tiene un gran impacto en la rentabilidad de la empresa [3].

Hoy en día, las empresas suelen administrar sus equipos utilizando un producto de software. Los sistemas de gestión de mantenimiento computarizado (CMMS, Computer Management Software Systems) son productos de software que ayudan a las empresas a ejecutar sus operaciones de mantenimiento de manera eficiente [4]. Con estos sistemas, las compañías pueden planificar el mantenimiento preventivo, administrar el mantenimiento correctivo, rastrear las horas de la máquina, los neumáticos y otros consumibles, monitorear los períodos de garantía, rastrear los costos de mano de obra y combustible y administrar el inventario. Los sistemas CMMS más avanzados se integran también con otras funciones de la empresa, tales como planificación de proyectos, recursos y contabilidad [5].

Hay muchos sistemas comerciales CMMS en el mercado. Sin embargo, algunas empresas tienen requisitos específicos para administrar sus equipos o desean una solución que pueda integrarse a su infraestructura de TI existente y otros sistemas operativos [6]. Para ellos, es importante la capacidad de personalizar y desarrollar aún más el sistema e integrarlo estrechamente a otras aplicaciones comerciales. Cada vez más, las empresas también buscan soluciones basadas en la nube. Los proveedores de soluciones que pueden ofrecer soluciones CMMS que cumplan con estos criterios pueden obtener una ventaja competitiva significativa en comparación con las soluciones puntuales o soluciones que requieren una instalación in situ.

Para aumentar la confiabilidad, la disminución en la frecuencia y la gravedad en las fallas de mantenimiento, y para favorecer la minimización de costos, control y seguimiento de procesos, dentro del Centro de Servicios y Gestión Empresarial (CESGE) del SENA Regional Antioquia [7] se desarrolló e implementó una herramienta de control de Mantenimiento que permite la participación de todos los miembros de la organización hacia la optimización y conservación de los equipos de infraestructura, tales como: aire acondicionado, luminarias, ascensores y reportes inmediatos de muebles, vidrios, ventanas, puertas, daños eléctricos, entre otros. La herramienta desarrollada implica un mejoramiento continuo y reducción de las necesidades del mantenimiento en el centro. Este trabajo describe el proceso de implementación del software, partiendo del concepto de mantenimiento, la metodología de desarrollo empleada, y como resultado final, descripción de las funcionalidades de la herramienta. Por último, se presentan algunas conclusiones sobre el proceso.

\section{LA GESTIÓN DEL MANTENIMIENTO EN EL CESGE}

En el entorno empresarial actual, las empresas deben buscar constantemente formas de obtener una ventaja competitiva. Muchas industrias requieren alta inversión en capital y cuando los costos de mantenimiento representan un porcentaje tan alto de los costos operativos totales, no es de extrañar que las compañías estén buscando formas de reducir los costos de mantenimiento [8]. Esto debe hacerse naturalmente sin sacrificar la confiabilidad del equipo o disminuir la calidad de los productos y servicios. Si se puede aumentar la efectividad del equipo, la confiabilidad o la productividad de la fuerza laboral o se puede reducir el uso de materiales, esto puede tener un impacto significativo en el resultado final de la empresa. Para muchas compañías, la implementación de programas de productividad de mantenimiento a menudo resulta en ahorros de $5 \%$ a $15 \%$ de los costos totales de mantenimiento [9].

Actualmente, la gestión de mantenimiento se define como la función empresarial que, a través de sus procesos de control, predicción, revisión, mejoramiento y reparación, permite garantizar el funcionamiento regular y el buen estado de conservación de los equipos e instalaciones [10]. Puede decirse que el principal objetivo del mantenimiento es asegurar que todo activo físico siga desempeñando las funciones determinadas. La gestión de mantenimiento debe contribuir a la mejora competitiva de la empresa, asegurando la disponibilidad y confiabilidad de los bienes, equipos e infraestructura de la compañía; la figura 1 describe los objetivos principales del mantenimiento. Un equipo o máquina con mal mantenimiento produce averías inoportunas y requiere tiempo adicional, para terminar los trabajos. Estos aumentos de tiempo extra magnifican los costos de operación [11]. 


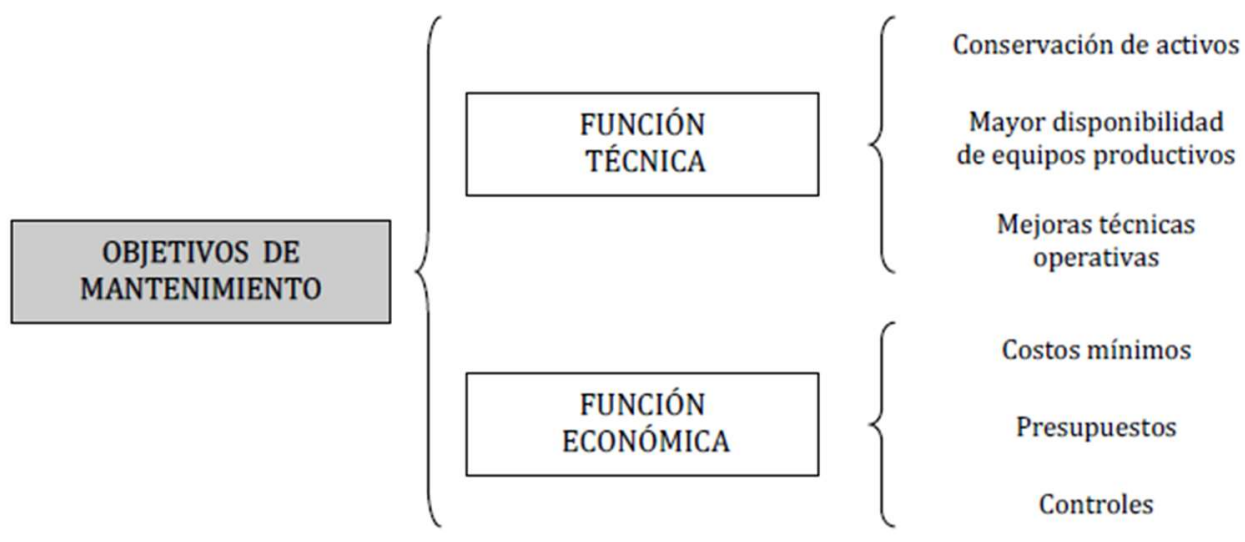

Figura 1. Objetivos del mantenimiento [12].

Por medio de un adecuado mantenimiento, se logra la reducción de fallas, que podrían ocasionar retrasos en las actividades de las empresas, además se maximiza el aprovechamiento de los equipos, muebles y enseres; la disponibilidad y durabilidad de estos últimos se garantiza, ya que se puede tener mejor administración y seguimiento de su uso y operación

\subsection{Sistemas de gestión de mantenimiento computarizado}

Los sistemas de gestión de mantenimiento computarizados son herramientas de software empaquetadas diseñadas específicamente para ayudar a las empresas en la gestión del mantenimiento. La mayoría de las empresas que mantienen equipos hoy en día tienen algún tipo de CMMS en uso y hay cientos de paquetes disponibles comercialmente para elegir. El crecimiento y la evolución en TI ha llevado a un aumento dramático en la capacidad y disponibilidad de herramientas de software para soportar el mantenimiento [13]. Esto también significa que los sistemas existentes pueden quedar obsoletos incluso en muy poco tiempo. Sin embargo, la tecnología no es un reemplazo para la estrategia y el software solo debe considerarse como un habilitador. La selección de software incorrecto, la implementación deficiente y los objetivos mal articulados a menudo conducen a fallas en los proyectos. Comprender la funcionalidad de CMMS, comparar diferentes opciones de software y alinearlas con los objetivos comerciales junto con el soporte de gestión son clave para implementaciones exitosas [14]. Por lo general, las empresas más pequeñas que utilizan soluciones especializadas están más satisfechas con sus CMMS. Las dos características más importantes para una implementación exitosa son la facilidad de adaptación a los procesos de mantenimiento y la facilidad de uso.

\subsection{Requerimientos de gestión de mantenimiento en el CESGE}

En el Centro de Servicios y Gestión Empresarial se venían realizando los procesos de mantenimiento de forma manual, lo cual se hacía engorroso. La falta de información registrada acerca de la ejecución de los servicios, dado que las actividades se ejecutaban solo de voz a voz, afectaba los procesos de auditorías, por lo cual se decidió agrupar todos los tipos de procesos en mantenimiento; por ejemplo, Aires acondicionados, Luminarias, Electricidad, ascensores y otros (muebles, vidrios, ventanas, puertas etc.). En este punto, comenzaron a realizar las solicitudes por correo electrónico, y a agendar y programar a los técnicos para dar solución a dichas solicitudes a través de dicho medio, pero la implementación del correo electrónico se vuelve compleja al momento de tener un control de los registros, ya que se debe manejar gran cantidad de información, y esta información se va acumulando.

Esta situación llevó a la propuesta de utilización de un software que permitiese la gestión de información de mantenimiento, dada la necesidad de agilizar y controlar estos procesos. Además, teniendo en cuenta que el CESGE dispone de una "Fábrica de software", un departamento con el talento humano y la infraestructura para desarrollar proyectos relacionados con Tecnologías de la Información y las Comunicaciones; se analizó el problema que se venía presentando y luego de un estudio de la situación surgió la idea de desarrollar SAM (Soluciones Agiles en Mantenimientos). SAM es una aplicación con la que se puede llevar un control y a la vez permite registrar a cualquier persona del centro de servicios, ya sean aprendices, personal administrativo, instructores, directivos o personal de oficios varios, entre otros perfiles, para realizar reporte de alguna novedad (daños, accidentes, mal funcionamiento de algún equipo entre otros) al administrador del área de mantenimiento del centro. Las novedades son recibidas y gestionadas a través del mismo SAM, el cual puede asignar técnicos en el sistema y llevar a cabo todo el proceso necesario para solucionar la necesidad planteada. 


\section{METODOLOGIA DE DISEÑO}

Para el desarrollo del aplicativo SAM se utilizó la metodología SCRUM, ya que es un proceso iterativo para controlar riesgo y optimizar la previsibilidad de un proyecto.[15], [16] basado en tres ejes fundamentales: Roles, Reuniones y Artefactos.

En primer lugar, se procede al levantamiento de requisitos con el cliente (en este caso los responsables de la gestión de mantenimiento), proceso que se realiza periódicamente y donde el cliente desempeña el rol de Product Owner, quien ofrece lineamientos acerca del "Qué" del producto, y adicionalmente hace la priorización de dichos requisitos, lo cual se convierte en el primer artefacto SCRUM como lo es el Product Backlog [17]. Con esta priorización se llega a la fase de planeación del primer Sprint; los sprint corresponden a la función de mayor prioridad respecto a la decisión del cliente, la cual se representa en una cartelera llamada Sprint Backlog, la cual contiene tres columnas (Por Hacer, en Proceso, Terminado) para ubicar las Historias de usuario (similares a los requerimientos pero en un lenguaje más accesible para el cliente). Tras la planeación de los Sprint iniciales, entra en acción el TEAM (Equipo de Desarrollo), encargado como tal del desarrollo del software. Una vez realizados de dos a tres Sprint se realiza el entregable llamado RELEASE, equivalente a una liberación de producto. En el caso de SAM se priorizó inicialmente la liberación de la funcionalidad de Aires Acondicionados para que los usuarios finales pudieran hacer las solicitudes de este ítem.

En pasos sucesivos se priorizó el mantenimiento de luminarias, temas eléctricos, otros (puertas, ventanas, vidrios, y otros). La metodología Scrum permite que el usuario final vaya trabajando con incrementos del producto mientras se va haciendo el desarrollo de la nueva funcionalidad, lo que da satisfacción al cliente pues no tiene que esperar un largo tiempo para tener módulos del aplicativo funcional [18]. Una vez hecho el "reléase" se requiere que el equipo haga una reunión llamada Retrospectiva, en donde tanto el equipo como el ScrumMaster (facilitador del proyecto, puente entre el Team y el Product Owner) puedan analizar las dificultades que hubo en la liberación; si hubo alguna estimación defectuosa de los Sprint (Sprint Review), se busca en las siguientes estimaciones lograr un avance del proyecto tomando las lecciones aprendidas, para así mejorar las estimaciones de forma que la gráfica de avance del proyecto (Burndown chart, ver figura 2) se alinee siempre al trazado óptimo, evidenciando que el proyecto se terminará con calidad en el tiempo estipulado [19].

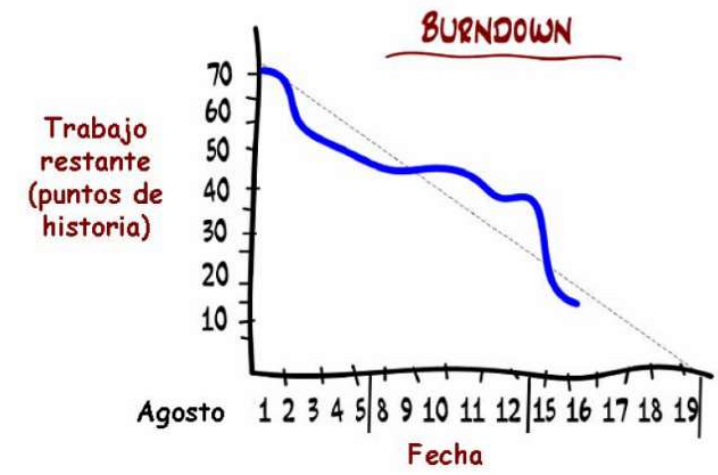

Figura 2. Gráfica de avance de proyecto (Burndown chart) según la metodología Scrum tomada de [20]

\section{DESCRIPCIÓN DEL SISTEMA SAM}

La figura 3 describe las fases del trabajo, y la herramienta de tecnología asociada a cada fase, en el desarrollo del aplicativo SAM [21]. El aplicativo comienza, en su pantalla de inicio, con el proceso que se encarga de administrar el ingreso a la aplicación mediante cuentas de usuario, tomando medidas de seguridad con respecto a los privilegios que se tiene según el rol de la cuenta. En esta aplicación se manejan dos roles de usuarios (Administrador y empleado).

En la figura 4 se muestra la vista de registro de solicitud para el mantenimiento de los equipos, en el cual se llena un formulario con la categoría del equipo afectado, indicando el lugar donde se debe atender el requerimiento, los datos de la persona que registra la solicitud con sus respectivos nombres, apellidos y correo electrónico de cuenta empresarial, y por último la descripción del daño ocurrido. A continuación, el menú inicio mostrará la barra lateral con todos los módulos del aplicativo, identificando el rol que el usuario ingresado tiene y las notificaciones que llegan, en el centro de la pantalla se muestran los paneles de las nuevas solicitudes: solicitudes para hoy, solicitudes atrasadas, técnicos disponibles, solicitudes sin asignar, y solicitudes atendidas. 


\begin{tabular}{|c|c|c|c|c|}
\hline $\begin{array}{l}\text { Fase del } \\
\text { desarrollo }\end{array}$ & Tecnología Asociada & Licenciamiento & versión & $\begin{array}{l}\text { Artefacto } y / 0 \\
\text { entregable. }\end{array}$ \\
\hline Requisitos & Microsoft (Excel) & Educativo & 365 & Matriz de requisitos \\
\hline Análisis & Enterprise Architect & Shreware & 8.0 .1 & Diagramas UML \\
\hline Diseño & $\begin{array}{l}\text { Uso de la arquitectura } \\
\text { MVC(Modelo, Vista, } \\
\text { Controlador) }\end{array}$ & No aplica & No aplica & Diagramas UML \\
\hline \multirow{5}{*}{ Desarrollo } & Framework - Mini-Master & GNU & $\begin{array}{l}\text { Versión } \\
\text { TINY }\end{array}$ & Código fuente PHP \\
\hline & PHP. & GNU & PHP 5.6 & $\begin{array}{l}\text { Código fuente PHP } \\
5.6\end{array}$ \\
\hline & JavaScript & GNU & $\begin{array}{l}\text { Java } \\
\text { Script } \\
\text { Safari }\end{array}$ & $\begin{array}{l}\text { Código Fuente Java } \\
\text { Script Safari }\end{array}$ \\
\hline & Ajax & GNU & Ajax 1.5 & $\begin{array}{l}\text { Código Fuente Ajax } \\
1.5\end{array}$ \\
\hline & Base de datos en MSQL & GNU & 5.0 .6 & Script Dd1 y Dml \\
\hline Despliegue & $\begin{array}{l}\text { Gestor de base de datos } \\
\text { en con servidor apache } \\
\text { Xampp }\end{array}$ & FREE & 5.6 .3 & $\begin{array}{l}\text { Script De Base De } \\
\text { Datos. }\end{array}$ \\
\hline
\end{tabular}

Figura 3. Fases del desarrollo del aplicativo web SAM.
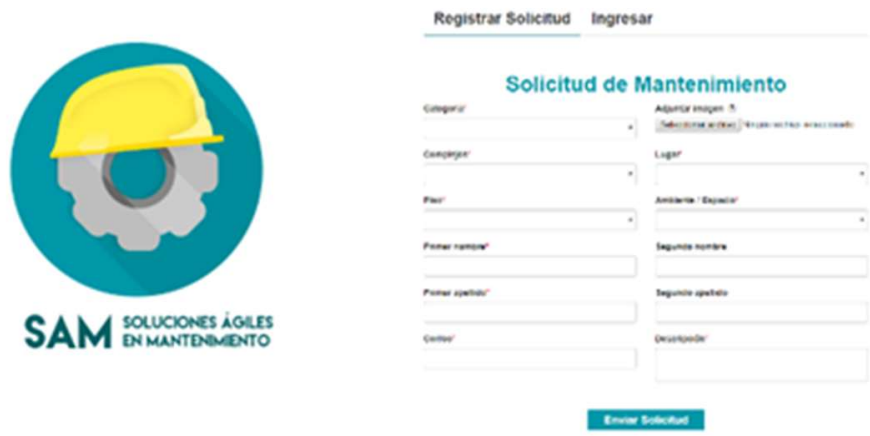

Figura 4. Captura de pantalla del menú de registro de solicitud.

El aplicativo permite la consulta de los usuarios que han sido creados previamente se puede visualizar de manera general todos los registros creados, por medio de una tabla interactiva que permite filtrar por diferentes categorías de datos. El registro de un nuevo usuario únicamente es posible desde la cuenta de administrador. Para consultar los equipos que han sido registrados previamente se pueden visualizar de manera general todos los registros creados, por medio de una tabla interactiva que permite filtrar por el código, marca o modelo del equipo; la figura 5 presenta una captura de pantalla del menú de equipos. El registro de un nuevo equipo sólo es posible desde la cuenta de administrador o técnico

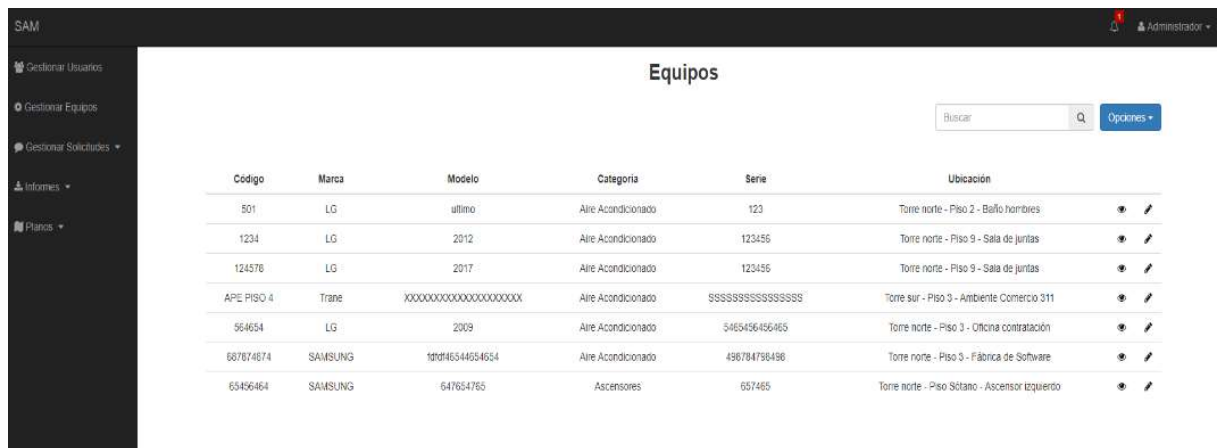

Figura 5. Captura de pantalla del menú de equipos.

Además, en el aplicativo SAM se puede consultar y hacer seguimiento a las solicitudes que han sido registradas previamente, pues permite visualizar de manera general todos los registros creados, por medio de una tabla interactiva que filtra por el estado, prioridad o ubicación geográfica; la tabla de consultar ofrece las opciones de ver el detalle, asignar los técnicos a las solicitudes registradas para realizar el mantenimiento y cambiar la prioridad de la solicitud (alta, media o baja). La figura 6 presenta una captura de pantalla del menú de solicitudes. 
Sin embargo, la asignación de un técnico a la solicitud solo es posible desde la cuenta de administrador.

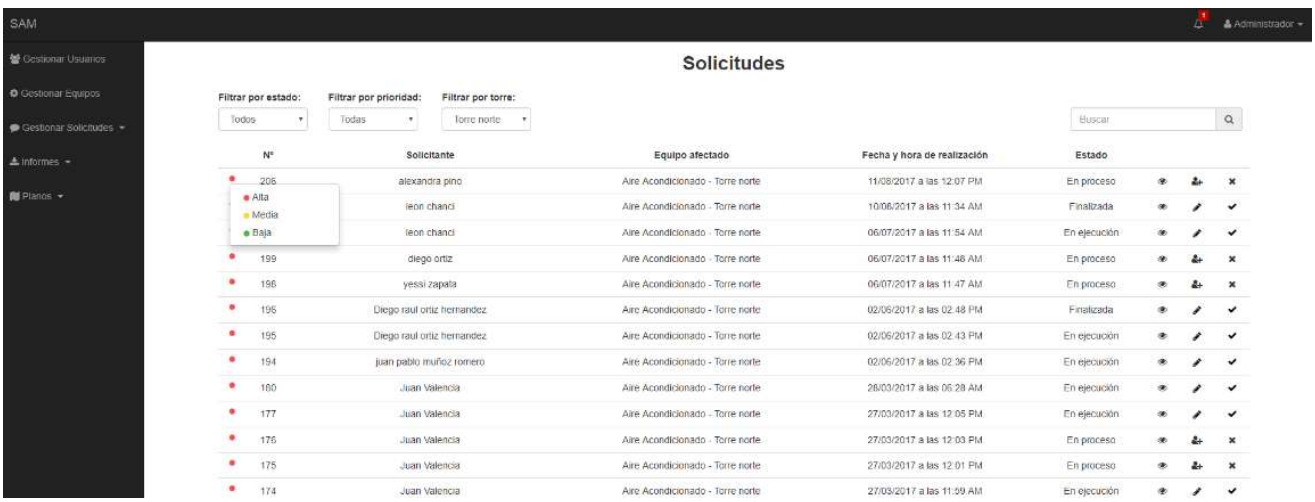

Figura 6. Captura de pantalla del menú de solicitudes

\subsection{Resultados preliminares del sistema SAM}

SAM se ha desarrollado para que en el Centro de Servicios y Gestión Empresarial se gestione el mantenimiento correctivo y preventivo de sus bienes y equipos, con base procesos ejecutados por medio de órdenes de trabajo, priorización de solicitudes, y el seguimiento y exportación de informes de los procedimientos que se han llevado a cabo. A través de SAM, en el CESGE Sena de la Regional Antioquia se ha podido llevar un control del mantenimiento de sus equipos, los responsables de la ejecución de los servicios (mantenimientos técnicos internos o externos), las programaciones de operaciones preventivas, y en general, de todos los elementos envueltos en el funcionamiento del departamento de mantenimiento. Según los estimados preliminares de las pruebas piloto, se han alcanzado reducciones hasta de un $40 \%$ en los tiempos que tarda una orden en ejecutarse, sin contar los ahorros en comunicación de información y en papel, al evitarse el uso de documentos impresos.

\section{CONCLUSIONES}

A través de la metodología puesta en práctica, se logró diseñar y desarrollar un aplicativo que permite minimizar los tiempos improductivos del personal, permitiendo al Centro de Servicios y Gestión Empresarial optimizar recursos humanos, económicos, ambientales y a la vez mejorar la atención de aquellos problemas y requerimientos que pueden prevenirse mediante un mantenimiento efectivo y adecuado. Cabe resaltar que este aplicativo aporta al desarrollo sostenible por medio de la minimización del papel, dado que a través de alertas y notificaciones en línea evita la impresión física de los formatos, simplifica las estadísticas, pues se pueden conocer con certeza las operaciones realizadas en un lapso determinado.

Actualmente, el uso de metodologías ágiles dentro del desarrollo de software permite que tanto cliente (Product Owner), el Facilitador del proyecto (Scrum Master) y el Equipo de desarrollo (Team), logren una verdadera sinergia para desarrollar un producto de calidad coherente con las expectativas de los usuarios finales, y que permita un adecuado retorno de la inversión para el cliente, produciendo valor y experiencia al Equipo, puesto que con cada incremento de producto (Sprint) se va reduciendo la incertidumbre y mejorando la precisión de sus estimaciones con respecto a tiempo y recursos necesarios en cada fase del ciclo de vida del software. El proyecto actual (SAM) es una muestra de ello.

\section{REFERENCIAS}

[1] E. Zapa Pérez, «Impacto de la Gestión por Procesos en la Innovación de las Organizaciones», Rev. CINTEX, vol. 19, pp. 23-37, 2014.

[2] P. Viveros, R. Stegmaier, F. Kristjanpoller, L. Barbera, y A. Crespo, «Propuesta de un modelo de gestión de mantenimiento y sus principales herramientas de apoyo Proposal of a maintenance management model and its main support tools», Rev. Chil. Ing., vol. 21, n. ${ }^{\circ}$ 1, pp. 125-138, 2012.

[3] J. Laurila, «Developing Computerized Maintenance Management System», Master of Engineering in Information Technology, Helsinki Metropolia University of Applied Sciences, Helsinki, 2017.

[4] M. I. Ardila Marín, W. Orozco Murillo, J. Galeano Echeverri, y A. M. Medina Escobar, «Desarrollo de software para la gestión del mantenimiento en los laboratorios de la I.U. Pascual Bravo», Rev. CINTEX, vol. 23, n. ${ }^{\circ}$ 1, pp. 43-50, oct. 2018.

[5] M. Wienker, K. Henderson, y J. Volkerts, «The Computerized Maintenance Management System an Essential Tool for World Class Maintenance», Procedia Eng., vol. 138, pp. 413-420, 2016. 
[6] M. D. Kent, O. Costello, S. Phelan, y K. Petrov, «Cost Oriented Maintenance Management Systems for Manufacturing Processes. ", IFAC-Pap., vol. 51, n. ${ }^{\circ}$ 30, pp. 48-53, 2018.

[7] J. J. Castro Maldonado, J. A. Patiño Murillo, y C. Gómez López, «Procesos de I+D+i en el Centro de Servicios y Gestión Empresarial del Servicio Nacional de Aprendizaje SENA 2015-2017», Rev. Espac., vol. 39, n. ${ }^{\circ} 20$, p. 21, 2018.

[8] D. Galar, A. Thaduri, M. Catelani, y L. Ciani, «Context awareness for maintenance decision making: A diagnosis and prognosis approach», Measurement, vol. 67, pp. 137-150, May 2015.

[9] S. Mostafa, S.-H. Lee, J. Dumrak, N. Chileshe, y H. Soltan, «Lean thinking for a maintenance process», Prod. Manuf. Res., vol. 3, n. ${ }^{\circ} 1$, pp. 236-272, ene. 2015.

[10] L. F. Jaramillo et al., «Modificación y Evaluación de un Lubricante con Nanotubos de Carbono para Aplicaciones en Sistemas Rodantes-Deslizantes», Rev. CINTEX, vol. 20, n. ${ }^{\circ}$ 2, pp. 9-33, 2015.

[11] G. García Monsalve, H. González S, y E. Cortés M, «Metodología De Mantenimiento Con Posible Aplicación En El Sector Agroindustrial», Rev. CES Med. Vet. Zootec., vol. 4, n. 2, pp. 137-150, 2009.

[12] W. Olarte, M. Botero, y B. Cañon, «IMPORTANCIA DEL MANTENIMIENTO INDUSTRIAL DENTRO DE LOS PROCESOS DE PRODUCCIÓN», Sci. Tech., vol. XVI, n. ${ }^{\circ} 44$, pp. 354-356, 2010.

[13] M. Macchi, L. Barberá Martínez, A. Crespo Márquez, L. Fumagalli, y M. Holgado Granados, «Value Assessment of e-Maintenance Platforms», en Advanced Maintenance Modelling for Asset Management, A. Crespo Márquez, V. González-Prida Díaz, y J. F. Gómez Fernández, Eds. Cham: Springer International Publishing, 2018, pp. 371-385.

[14] F. Hoyos Gómez, J. D. Betancur Gómez, D. Osorio Patiño, y J. G. Ardila Marín, «Construcción de curvas de factor de concentración de esfuerzos por medio de simulaciones», Rev. CINTEX, vol. 21, n. ${ }^{\circ} 1$, jun. 2016.

[15] H. Lei, F. Ganjeizadeh, P. K. Jayachandran, y P. Ozcan, «A statistical analysis of the effects of Scrum and Kanban on software development projects», Robot. Comput. -Integr. Manuf., vol. 43, pp. 59-67, 2017.

[16] N. E. Viana-Rúa, L. Y. Arenas-Becerra, y J. A. Patiño-Murillo, «Development of an interactive tool for soft skills enhancement in people with mild cognitive disabilities», J. Phys. Conf. Ser., vol. 1418, p. 012003, dic. 2019.

[17] A. Peralta, «Universidad ORT Uruguay». p. 12, 2005.

[18] J. J. Castro-Maldonado, J. A. Londoño-Gallego, S. Londoño-Marin, y J. A. Patiño-Murillo, «Implementation of a technological, information, and communication tool for project management in the network of Tecnoparque, Colombia», J. Phys. Conf. Ser., vol. 1418, p. 012014, dic. 2019.

[19] S. Londoño Marín, J. A. Londoño Gallego, J. J. Castro Maldonado, y J. A. Patiño Murillo, «Guía interactiva para el desarrollo de proyectos bajo la Metodología de Marco Lógico», Rev. Espac., vol. 39, n. ${ }^{\circ}$ 47, p. 25, 2018.

[20] H. Kniberg, Scrum and XP desde las trincheras. 2007.

[21] A. Peralta y F. P. Romero, «Toma de Decisiones a partir de Conocimiento Extraído tras el Análisis de Comportamientos Previos. Aplicación Práctica para la Dirección de Proyectos de Desarrollo de Software», Rev. CINTEX, vol. 20, n. ${ }^{\circ}$ 2, pp. 97-111, dic. 2015. 